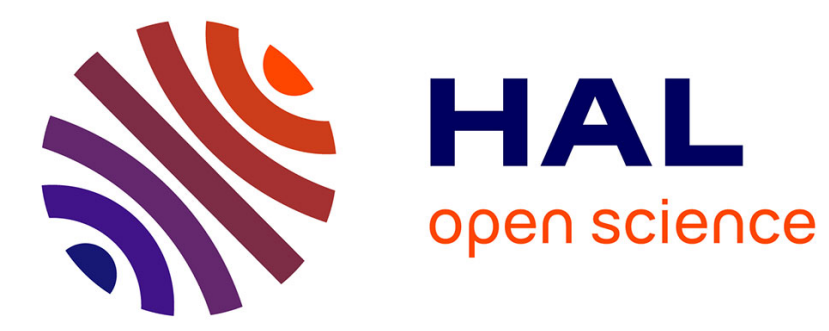

\title{
Lock-in thermo-electric detector arrays: thermal cross-talk prediction by non-linear model
}

W Vandermeiren, J Stiens, G Shkerdin, C de Tandt, R Vounckx

\section{To cite this version:}

W Vandermeiren, J Stiens, G Shkerdin, C de Tandt, R Vounckx. Lock-in thermo-electric detector arrays: thermal cross-talk prediction by non-linear model. Journal of Physics D: Applied Physics, 2011, 44 (5), pp.55101. 10.1088/0022-3727/44/5/055101 . hal-00591291

\section{HAL Id: hal-00591291 https://hal.science/hal-00591291}

Submitted on 9 May 2011

HAL is a multi-disciplinary open access archive for the deposit and dissemination of scientific research documents, whether they are published or not. The documents may come from teaching and research institutions in France or abroad, or from public or private research centers.
L'archive ouverte pluridisciplinaire $\mathbf{H A L}$, est destinée au dépôt et à la diffusion de documents scientifiques de niveau recherche, publiés ou non, émanant des établissements d'enseignement et de recherche français ou étrangers, des laboratoires publics ou privés. 


\title{
Lock-in thermo-electric detector arrays: thermal cross-talk prediction by non-linear model
}

\author{
W. Vandermeiren ${ }^{\mathrm{a}}$, J. Stiens ${ }^{\mathrm{a}}$, G. Shkerdin ${ }^{b}$, C. De Tandt ${ }^{\mathrm{a}}$ and R. Vounckx ${ }^{\mathrm{a}}$ \\ ${ }^{a}$ Laboratory for Micro- and Photonelectronics, ETRO-FirW \\ VUB, Pleinlaan 2, B-1050 Brussel, Belgium \\ ${ }^{b}$ Institute of Radio Engineering and Electronics of the Russian Academy of Science \\ Vvedenskogo Square 1, 141120 Fryazino (Moscow reg.), Russia \\ E-mail: wvanderm@etro.vub.ac.be
}

\begin{abstract}
A non-linear numerical finite element method (FEM) model of a thermo-electric focal plane array (FPA) detector is presented here. Laser induced thermo-voltage profiles tend to spread out for small lock-in frequencies as the thermal diffusion length is inversely proportional to the square-root of the lock-in frequency. This leads to a frequency and spatial dependent thermal cross-talk level. In this paper we investigate the thermal cross-talk level quantitatively in function of spatial coordinates and lock-in frequency. Experimental data are provided at an optical power level of $1 \mathrm{~W}$. The impact of non-linear thermal parameters as the temperature dependence of the absorption coefficient, the thermal conductivity, the heat transfer coefficient and the Seebeck coefficient on the thermal profile and cross-talk level generated inside the detector material are studied in detail. Heat losses that are included in the model are conduction and laminar free convection. The relative importance of the above mentioned non-linear thermal parameters in terms of thermal cross-talk for steady-state solutions are discussed as well.
\end{abstract}

Keywords: Thermo-electric detector, FEM model, non-linear thermal modeling

\section{Introduction}

Lock-in thermography greatly improves the sensitivity and the image resolution as compared to steady-state thermography [1]. The latter one suffers from high lateral heat spreading which results in a poor resolution. In our study, a similar lock-in technique is applied on Seebeck focal plane array's (FPA's) to improve the resolution (pixel density) and/or thermal cross-talk performance. In contrast to lock-in thermography, the thermal profile is probed by the Seebeck effect, by means of electrodes, instead of a contactless IR camera measurement.

Lock-in thermo-electric FPA detectors can be used for laser beam profilometry [2]. Partial absorption of high power laser beams can result in a temperature distribution with local surface temperature maxima which exceeds $100 \mathrm{~K}$ above room temperature. In this temperature range, some non-linear thermal parameters (e.g. the thermal conductivity of GaAs) will change significantly with respect to its constant property-value. Hence, most relevant non-linear thermal parameters should be taken into account for accurate modeling. This paper is concerned with thermal cross-talk reduction in function of spatial coordinates and lock-in frequency. The impact of the temperature dependence of the absorption coefficient, the thermal conductivity, the heat transfer coefficient and the Seebeck coefficient, further referenced as non-linear thermal parameters, on the thermal cross-talk level in function of the applied lock-in frequency are investigated. 
Note that when non-linearities are ignored the thermal profile might be off by 25 degrees for a temperature increase of the order of $100 \mathrm{~K}$. The non-linear FEM model enables to predict the laser induced thermal profiles more accurately as compared to a linear model. It might be important to accurately simulate the thermal profiles to define save operation conditions to avoid any damage as a consequence of local thermal runaway. It has been shown that the absolute temperature may strongly affect the reliability and performance of semiconductor based devices [3].

In a first section, we describe the numerical FEM model which was used to calculate the laser induced spatio-temporal temperature distribution inside the active detector material. The results of the numerical calculations are shown and discussed in the second section. The final conclusions are drawn in a third section.

\section{The numerical FEM model}

The numerical FEM model geometry, used to simulate the laser induced spatio-temporal temperature or thermo-voltage distribution, is illustrated in figure 1. The simulation of the thermal profile involves solving the heat equation. This is a partial differential equation (PDE) with boundary conditions which is solved in four dimensions (spatial coordinates and time). Heat transfer by free convection - which is assumed to be the main cooling mechanism of the system for steady state solutions - is proportional to the external area. Hence, accurate temperature profile simulations at steady state condition can only be realized by modeling the complete thermal system, including the open cavity package (OCP) in which the detector is placed as it significantly contributes to the external area of the system (heat sink). Hence, an advanced thermal model was developed in "Comsol Multiphysics" in which only $1 / 4$ of the total structure is simulated due to symmetry considerations.

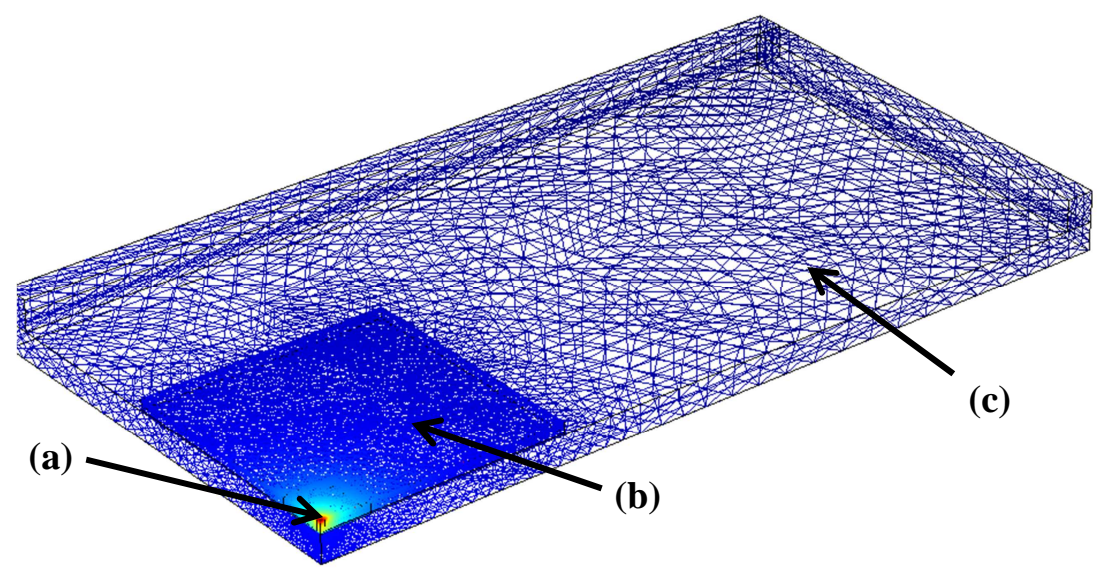

Figure 1. Thermal FEM model of a Seebeck FPA detector; (a) heat center; (b) detector substrate (GaAs); (c) open cavity package.

The model consists of a $350 \mu \mathrm{m}$ thick undoped GaAs substrate with a thin $(2 \mu \mathrm{m})$ highly doped absorbing layer $\left(\mathrm{N}_{\mathrm{D}}=4 \times 10^{18} \mathrm{~cm}^{-3}\right)$ at the top surface of the detector. The non-ideal thermal contact between the substrate and the stainless steel OCP is modeled by means of a thin thermal grease layer with a thermal conductivity of $0.7 \mathrm{Wm}^{-1} \mathrm{~K}^{-1}$. The heat conduction equation, given by Eq.1, is then solved in three dimensions.

$$
Q_{H S}=\rho C_{p} \frac{\partial T}{\partial t}-\nabla\left(k_{t h} \nabla T\right)
$$

Here, $\mathrm{Q}_{\mathrm{HS}}, \mathrm{T}, \mathrm{C}_{\mathrm{p}}, \rho$ and $\mathrm{k}_{\mathrm{th}}$ are the heat source, the temperature, the heat capacity, the density and the thermal conductivity, respectively. Free convection to air is assumed at all external boundaries. Eq. 2 expresses the boundary condition with $\mathrm{k}_{\mathrm{th}}, \mathrm{h}, \mathrm{T}$ and $\mathrm{T}_{\mathrm{inf}}$ the thermal conductivity, the heat transfer coefficient, the temperature and the external temperature, respectively.

$$
k_{t h} \nabla T=h\left(T_{i n f}-T\right)
$$


All numerical calculations were performed for a $\mathrm{CO}_{2}$ laser emitting at its standard wavelength $\lambda=10.6 \mu \mathrm{m}$. The thermo-voltage is measured at the top surface of the GaAs substrate with the average temperature at the backside of the substrate as a reference. The absorption of the incident laser beam is modeled as a heat source term $\mathrm{Q}_{\mathrm{CW}}$ described by Eq. 3,45 and 6 for a single pass through the absorption layer.

$$
\begin{aligned}
Q_{C W}= & \left(1-F R\left(n_{\text {air }}, n_{G a A s}\right)\right) \cdot\left|\frac{\partial I(x, y, z)}{\partial z}\right| \cdot A R(z) \quad\left(W m^{-3}\right) \\
\text { With } \quad & I(x, y, z)=I_{0} \cdot \exp \left(-2\left(\frac{x^{2}+y^{2}}{w^{2}}\right)\right) \cdot \exp \left(\alpha_{e} z\right) \quad \text { for } z \leq 0 \\
& A R(z)=\left(z \geq-T_{A L}\right) \\
& F R\left(n_{\text {air }}, n_{\text {GaAs }}\right)=\left(\frac{n_{G a A s}-n_{\text {air }}}{n_{G a A s}+n_{\text {air }}}\right)^{2}
\end{aligned}
$$

Here, $\mathrm{z}=0$ corresponds to the top surface of the GaAs specimen. The heat source term $Q_{C W}$ is proportional to the change in intensity $\left|\frac{\partial I(x, y, z)}{\partial z}\right|$ along the axis of light propagation induced by the absorption process. As a consequence, $Q_{C W}$ is proportional to the lateral laser intensity profile and the absorption coefficient $\alpha_{\mathrm{e}}$. AR(z), given by expression 5 is a binary function which limits the absorption region to the absorption layer thickness $\mathrm{T}_{\mathrm{AL}}$. The heat source takes the Fresnel reflection $\left(\mathrm{FR}\left(\mathrm{n}_{\text {air }}, \mathrm{n}_{\mathrm{GaAs}}\right)\right)$ into account at the top surface of the substrate with $n_{\text {air }}$ and $n_{\text {GaAs }}$, the refractive index of air and GaAs, respectively. Double propagation of light through the absorption layer is taken into account as the retaining intensity after one time pass is back reflected by a metallization layer at the bottom on the substrate. This typically results in a dissipation of the order of $15 \%$ of the incident optical power in our designs.

\section{Non-linear thermal parameters}

The temperature dependence of the absorption coefficient of $10.6 \mu \mathrm{m}$ in $\mathrm{n}-\mathrm{GaAs}$ with a doping concentration of $4 \times 10^{18} \mathrm{~cm}^{-3}$ was calculated based on a multi-valley $(\Gamma, \mathrm{X}, \mathrm{L})$ model where non-parabolic and anisotropic effects were taken into account for the intra-valley and (non)equivalent inter-valley absorption mechanisms [4,5]. A general expression for the total absorption coefficient $\alpha_{e}$ by $\Gamma, \mathrm{L}$ and $\mathrm{X}$-electrons is given by

$$
\begin{gathered}
\alpha_{e}=2 \frac{\omega}{c} \operatorname{Im}\left(\sqrt{\varepsilon_{e}(\omega)}\right) \\
\text { With } \quad \varepsilon_{e}(\omega)=\varepsilon_{1}\left(1-\frac{\omega_{p, \Gamma}^{2}+\omega_{p, \mathrm{~L}}^{2}+\omega_{p, \mathrm{X}}^{2}}{\omega^{2}}+i\left(\frac{\omega_{p, \Gamma}^{2}}{\omega^{3} \tau_{\Gamma}}+\frac{\omega_{p, \mathrm{~L}}^{2}}{\omega^{3} \tau_{L}}+\frac{\omega_{p, \mathrm{X}}^{2}}{\omega^{3} \tau_{X}}\right)\right)
\end{gathered}
$$

Where $\omega_{p, \Gamma, \mathrm{L}, \mathrm{X}}=\sqrt{4 \pi n_{\Gamma, \mathrm{L}, \mathrm{X}} e^{2} /\left(m_{\Gamma, \mathrm{L}, \mathrm{X}} \varepsilon_{1}\right)}, n_{\Gamma, \mathrm{L}, \mathrm{X}}$ and $m_{\Gamma, \mathrm{L}, \mathrm{X}}$ are the plasma frequency, the electron concentration and optical effective mass of the $\Gamma$, L- and X-valley electrons, respectively, $\varepsilon_{1}$ is the permittivity of undoped GaAs at medium infrared wavelengths and $\omega$ is the electromagnetic wave angular frequency. The values $\tau_{\Gamma, \mathrm{L}, \mathrm{X}}$ are directly connected with the absorption coefficients by $\Gamma, \mathrm{L}-$ and $\mathrm{X}$ - valley electrons. Figure 2 shows the temperature dependent absorption coefficient used in the thermal FEM model. A good agreement between the model and experimental data can be achieved if the compensation factor given by $F_{c o m p}=\frac{N_{D}+N_{A}}{N_{D}-N_{A}}$ is known, where $\mathrm{N}_{\mathrm{D}}$ and $\mathrm{N}_{\mathrm{A}}$ are the donor and 
acceptor concentration, respectively. A compensation factor of one is assumed in our model such that the contribution of impurity scattering on compensated donor and acceptor atoms to the absorption coefficient can be neglected.

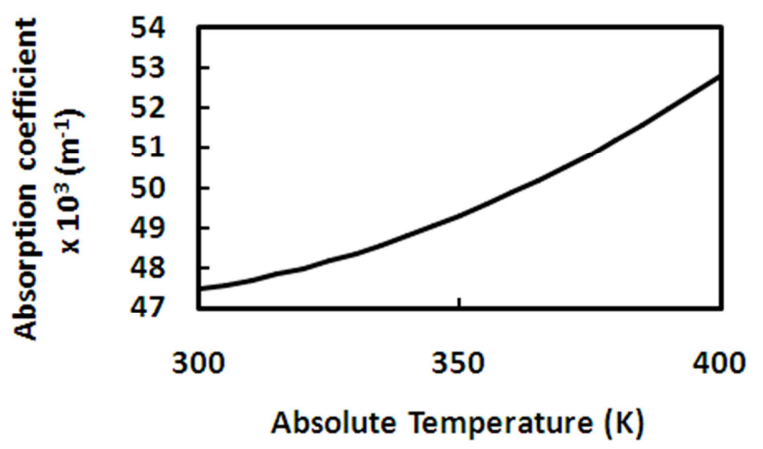

Figure 2. Absorption coefficient for $10.6 \mu \mathrm{m}$ wavelength in $\mathrm{n}-\mathrm{GaAs}$ with $\mathrm{N}_{\mathrm{D}}=4 \times 10^{18} \mathrm{~cm}^{-3}$.

A second order polynomial curve fit on this data results in an analytical expression (Eq. 9) for the absorption coefficient which is valid within a temperature range of $300 \mathrm{~K}$ to $400 \mathrm{~K}$. Notice that the absorption coefficient increases about $11.4 \%$ in this temperature interval.

$$
\alpha_{e}(T)=0.34 T^{2}-183.3 T+72030 \quad\left(m^{-1}\right)
$$

The thermal conductivity is even more sensitive to the absolute temperature. It varies roughly between 45 and $30 \mathrm{Wm}^{-1} \mathrm{~K}^{-1}$ in the temperature range of $300 \mathrm{~K}$ to $400 \mathrm{~K}[6,7,14]$. A linear approximation of the temperature dependence of the thermal conductivity, given by Eq. 10, is used in our model.

$$
k_{t h}(T)=45-0.15(T-300) \quad\left(W m^{-1} K^{-1}\right)
$$

The temperature dependence of the heat capacity and the density of n-GaAs are neglected in our model for the temperature range under consideration. The Seebeck coefficient has been calculated by solving the kinetic equation for the electron distribution function in the presence of an electron temperature gradient. Taking into account the non-parabolicity of the $\Gamma$-valley and the anisotropy of the satellite L- and $\mathrm{X}$-valleys of $\mathrm{n}-\mathrm{GaAs}$, the expression for $\mathrm{S}$ can be written as [8]

$$
S=\frac{k_{B}\left(a_{\Gamma} \sigma_{\Gamma}+a_{\mathrm{L}} \sigma_{\mathrm{L}}+a_{\mathrm{x}} \sigma_{\mathrm{x}}\right)}{\left(\sigma_{\Gamma} \cdot \sigma_{\mathrm{L}} \cdot \sigma_{\mathrm{x}}\right)}
$$

Where $\mathrm{k}_{\mathrm{B}}$ and $\mathrm{q}$ are the Boltzmann constant and the elementary charge respectively; $\sigma_{\Gamma, \mathrm{L}, \mathrm{X}}$ and $\mathrm{a}_{\Gamma, \mathrm{L}, \mathrm{X}}$ are the electron conductivity and the dimensionless weighting coefficients for the $\Gamma, \mathrm{L}$ and $\mathrm{X}$ valley, accordingly. The temperature dependence of the Seebeck coefficient for n-GaAs with a doping density of $\mathrm{N}_{\mathrm{D}}=4 \times 10^{18} \mathrm{~cm}^{-3}$ is then approximated by

$$
S(T)=86\left(1.3+3.45 \cdot 10^{-3}(T-300)\right) \quad\left(\mu V K^{-1}\right)
$$

The contribution of the phonon-drag effect to the thermo-electric power is neglected as the active semiconductor layer is degenerate and the operation temperature is relatively high $\mathrm{T} \geq 297 \mathrm{~K}$ [9]. Heat transfer by free convection is characterized by a heat transfer coefficient $\mathrm{h}$ of the order of $10 \mathrm{Wm}^{-2} \mathrm{~K}^{-1}$. Although, often approximated by a constant value, this coefficient can differ substantially from its constant-property value in function of temperature. The temperature dependent viscosity and density can affect the velocity profile significantly [10] and will consequently contribute to the temperature dependence of the heat transfer coefficient. The temperature dependence of the heat transfer coefficient was calculated, assuming that natural convection at the boundaries of the detector structure could be modeled by a laminar air flow on a vertical plate, taking into account the temperature 
dependence of the viscosity and density of air [11]. The heat transfer coefficient for free convection on a vertical plate in air is given by [11]

$$
h(T)=N u \cdot k_{\text {th,air }} \cdot(H)^{-1} \quad\left(W m^{-2} K^{-1}\right)
$$

with $\mathrm{k}_{\text {theair }}$ and $\mathrm{H}$, the thermal conductivity of air and the vertical plate height, respectively. The Nusselt number is expressed as [11]

$$
N u(T)=0.65+\frac{0.67 R a^{1 / 4}}{\left[1+(0.492 / P r)^{9 / 16}\right]^{8 / 27}}
$$

Where Pr is the dimensionless Prandtl number of the order of 0.7 and Ra is the Rayleigh number. The latter one was calculated taken into account the average surface temperature of the model in contact with air, rather than using the local temperature, as the empirical formulation for the Nusselt number is only valid for isothermal plates. Consequently, the heat transfer coefficient is considered equal at all boundaries such that it will not influence the lateral thermal cross-talk level. However, it will influence the thermal wave amplitude as it varies over time due to average surface temperature fluctuations. This approximation will result in an over-estimation of the non-linearity induced thermal cross-talk reduction. However, local heat transfer coefficients can be estimated by solving an inverse problem as proposed by M. Janicki [12], although not done in this paper. The temperature dependence of the heat transfer coefficient is illustrated in figure 3. A good agreement between such a model and experimental data can be achieved as shown by G. Hanreich [16].

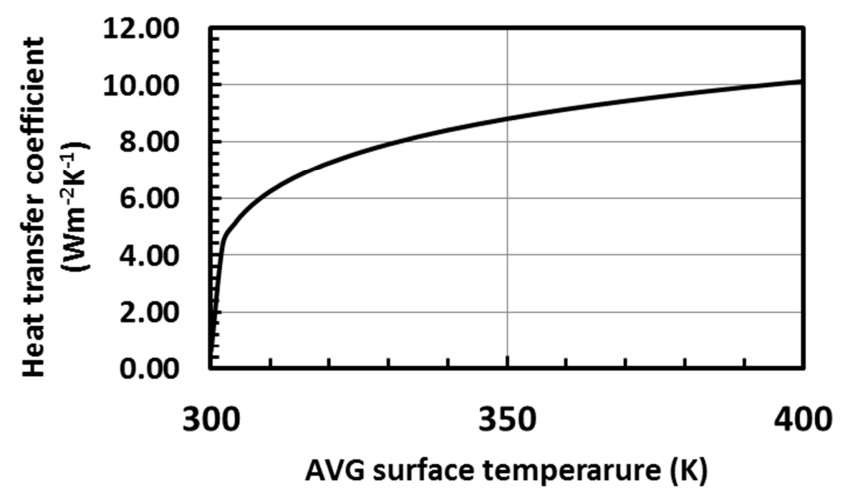

Figure 3. Temperature dependence of the heat transfer coefficient.

\section{Simulations and discussion}

This section of the paper presents the numerical simulations in according to the above described model. The simulations are carried out for a modulated Gaussian shaped $\mathrm{CO}_{2}$ laser beam with a fixed beam diameter of $250 \mu \mathrm{m}$ and a power level of $2 \mathrm{~W}$ to $10 \mathrm{~W}$. The time dependent heat source term becomes

$$
Q(t)=\frac{1}{2}\left(1+\sin \left(2 \pi f_{\text {lock-in }} t\right)\right) \cdot Q_{C W} \quad\left(W m^{-3}\right)
$$

Where $\mathrm{f}_{\text {lock-in }}$ is the modulation frequency and $\mathrm{Q}_{\mathrm{cw}}$ is the heat source term corresponding to continuous wave illumination (see Eq. 3). Note that the average incident power level is half of the continuous wave power. Hence, a steady state solution of half power was used as an initial condition for the time dependent simulation such that the thermal equilibrium condition was established from the start. The time dependent heat conduction equation was then solved in three dimensions for optical chopper frequencies going from CW (continuous wave) to $500 \mathrm{~Hz}$. The simulation output is a time dependent $3 \mathrm{D}$ thermal distribution for each of these frequencies. The thermal distribution is subsequently converted into a thermo-voltage distribution by means of relation 8 (specified for $\mathrm{N}_{\mathrm{D}}=4 \times 10^{18} \mathrm{~cm}^{-3}$ within the range $300 \mathrm{~K}<\mathrm{T}<400 \mathrm{~K}$ ). As a reference, the average temperature at the backside of the 
GaAs substrate was considered. Figure 4 shows the temperature distribution at the top surface of the specimen over a lateral distance of $8 \mathrm{~mm}$ from the heat center for different modulation frequencies.

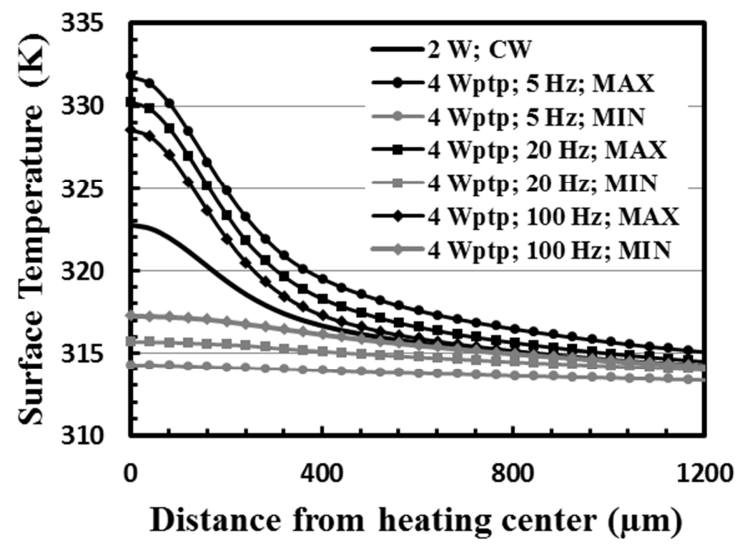

Figure 4. Surface temperature distribution for different modulation frequencies.

The thermal wave amplitude (MAX - MIN) decreases symmetrically around the CW thermal profile as the modulation frequency increases. Furthermore, we notice a decrease in amplitude for an increasing distance from the heating center due to the frequency limited thermal diffusion length. An analytical expression for the thermal diffusion length in the case of a point-like heat source is given by [13]

$$
\mu_{t h}\left(f_{\text {lock-in }}\right)=\sqrt{\frac{\alpha_{\text {th,GaAs }}(T)}{\pi f_{\text {lock-in }}}} \quad(m)
$$

Where $\alpha_{\mathrm{th}}, \mathrm{GaAs}$ and $\mathrm{f}_{\text {lock-in }}$ are the thermal diffusivity and lock-in frequency, respectively. The periodic temperature increase at a distance $r$ from the point-like heat source is then described by

$$
\partial T(r)=\frac{Q}{4 \pi k_{t h} r} \cdot \exp \left(-(1+i) \frac{r}{\mu_{t h}}\right)
$$

Where $\mathrm{Q}, \mathrm{k}, \mu$ and $\mathrm{r}$ are the power, thermal conductivity, thermal diffusion length and the distance from the heat source. Hence, the periodic temperature increase is proportional to $\exp \left(-\sqrt{f_{\text {lock-in }}}\right)$. By means of Eq. 17 one can analytically extract the cross-talk reduction factor for GaAs. Figure 5 shows the cross-talk reduction level in $\mathrm{dB}$ in function of the lock-in frequency at a distance of $400 \mu \mathrm{m}$ (upper curves) and $800 \mu \mathrm{m}$ (lower curves) from the heating center for an incident laser power of $1 \mathrm{~W}$. A good agreement was found between experimental data (solid black curve), the numerical FEM model (dashed curve) and the analytical model as described above. For detailed information about the experimental setup, we refer to [2]. Note that relatively high thermal cross-talk reduction factors are achieved for modest lock-in frequencies. E.g., at a modulation frequency of $500 \mathrm{~Hz}$, a cross-talk reduction factor of $-34 \mathrm{~dB}$ is realized at $400 \mu \mathrm{m}$ from the heating center. One can also conclude that the highest gain in terms of thermal cross-talk reduction per $\mathrm{Hz}$ is realized at low lock-in frequencies. 


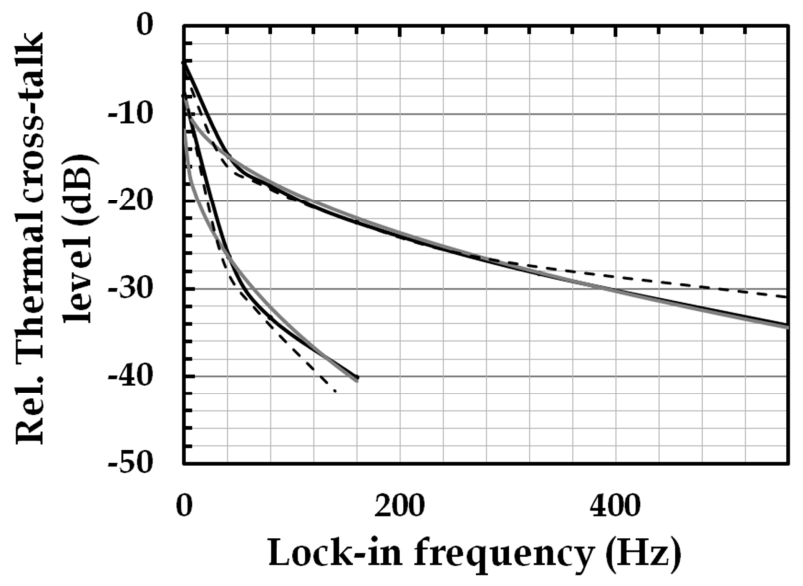

Figure 5. Thermal cross-talk reduction (dB) versus lock-in frequency at $400 \mu \mathrm{m}$ and $800 \mu \mathrm{m}$ from the heating center $\left(\mathrm{P}_{\mathrm{opt}}=1 \mathrm{~W}\right)$.

Non-linear effects were investigated by varying the incident optical power level $\left(\mathrm{P}_{\mathrm{opt}}\right)$ from $2 \mathrm{~W}$ to $8 \mathrm{~W}$ for a fixed laser beam width of $250 \mu \mathrm{m}$. At $\mathrm{P}_{\mathrm{opt}}=8 \mathrm{~W}$, a maximum absolute surface temperature of about $400 \mathrm{~K}$ was found which is at the border of the validity interval of most of the previously discussed non-linear parameter approximations. Figure 6 shows the thermo-voltage and thermal cross-talk profile for different incident power levels. Here, the solid black curve corresponds to the incident optical intensity profile. The light grey curves are the normalized thermo-voltage profiles for different optical power levels and the dark gray lines are the corresponding thermal cross-talk levels. In these simulations, the thermal cross-talk level is defined as any relative deviation of the normalized thermo-voltage profile, referenced to the average temperature at the backside of the specimen, with respect to the normalized incident intensity profile.

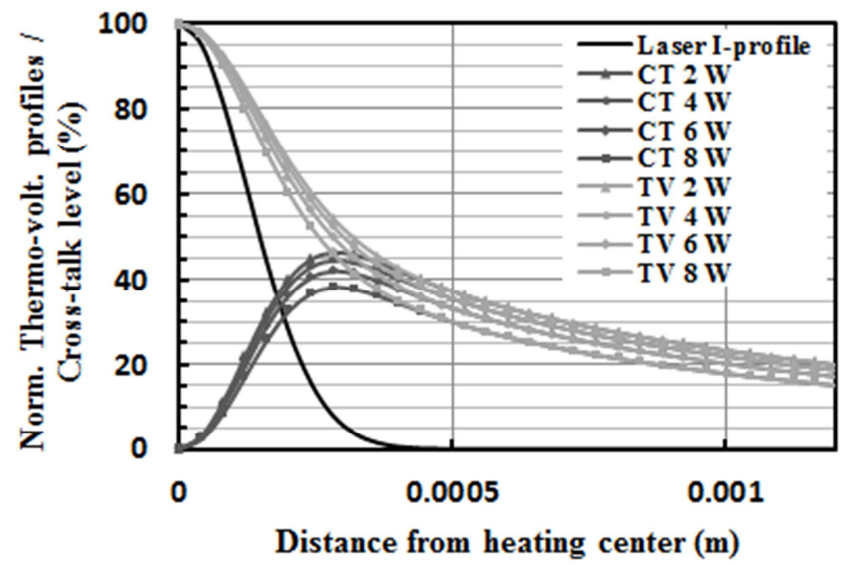

Figure 6. Thermo-voltage and thermal cross-talk profile for different power levels (CW illumination).

The maximum impact of the non-linearities on the thermal cross-talk level is found at a distance of the order of the laser beam size. Our simulation shows a non-linearity assisted cross-talk reduction of about $8 \%$ for $\mathrm{CW}$ illumination and $\mathrm{P}_{\text {opt }}$ between $2 \mathrm{~W}$ and $8 \mathrm{~W}$. Note that this is a conservative measure as the reference temperature is also subjected to these non-linear effects. In case of a fixed $300 \mathrm{~K}$ reference, one obtains a thermal cross-talk reduction of about $15 \%$ for the same power range. 


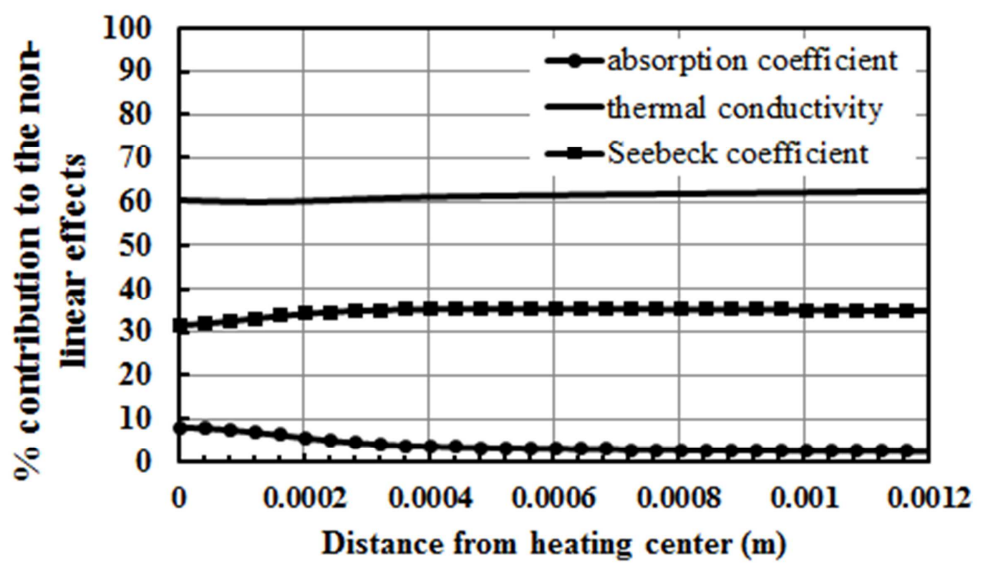

Figure 7. Relative contribution of non-linear thermal parameters to the cross-talk level at CW illumination.

The relative contributions of the thermal non-linearity assisted cross-talk reduction have been investigated at continuous wave illumination for the power range of $2 \mathrm{~W}$ to $8 \mathrm{~W}$. Figure 7 shows that the main contribution (about $60 \%$ ) can be assigned to the temperature dependence of the thermal conductivity. The non-linear Seebeck coefficient and absorption coefficient are responsible for about $30 \%$ and $10 \%$ of the overall thermal non-linearity assisted cross-talk reduction. Note that, although, the Seebeck coefficient doesn't influence the temperature distribution inside the detector material, the non-linear behavior of the coefficient is in favor of the cross-talk reduction and contributes significantly to the total cross-talk reduction of the detector.

Figure 8 shows the thermal cross-talk level in function the lock-in frequency for different incident power levels. One sees that the impact of the non-linear thermal parameters vanishes as the lock-in frequency increases. Concretely, the simulations show that the impact of the thermal non-linearities on the thermal cross-talk level reduces from about $5 \%$ at $5 \mathrm{~Hz}$ to about $0.5 \%$ at $500 \mathrm{~Hz}$.

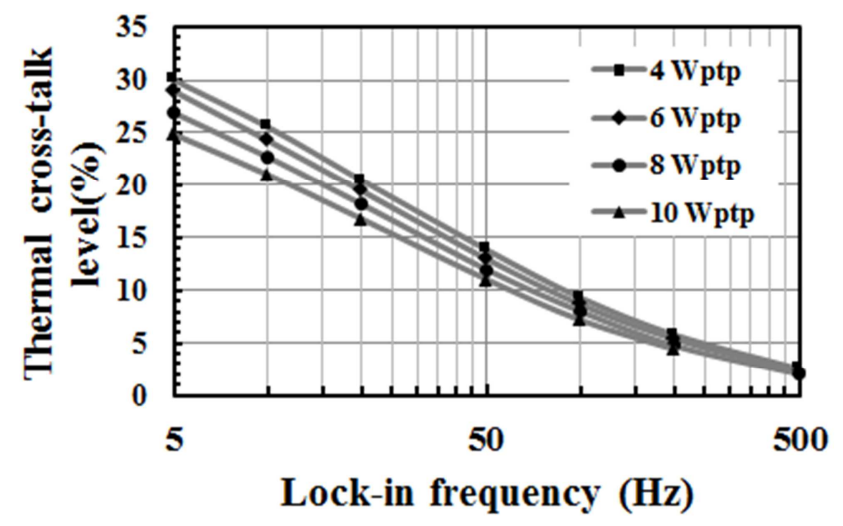

Figure 8. Frequency dependence of the thermal cross-talk level for different lock-in frequencies.

\section{Conclusion}

A non-linear FEM model of a lock-in thermo-electric FPA detector was presented. The heat equation was solved in three dimensions (geometry of the detector) in function of time which resulted in a laser induced spatio-temporal thermal profile. Quantitative simulations were carried out for different lock-in frequencies and optical power levels. It was shown that the thermal cross-talk level can be considerably reduced by increasing the lock-in frequency. For example, at a modulation frequency of $500 \mathrm{~Hz}$, a cross-talk reduction factor of $-34 \mathrm{~dB}$ is realized at $400 \mu \mathrm{m}$ from the heating center. Numerical simulations at a power level of $1 \mathrm{~W}$ where compared to experimental data and an analytical model and a good agreement was found. One can also conclude that the highest gain in terms of thermal cross-talk reduction per $\mathrm{Hz}$ is realized at low lock-in frequencies. 
Furthermore we investigated the impact of non-linear thermal parameters in terms of cross-talk reduction by performing simulations at different power levels. The impact of the non-linear parameters on the cross-talk level was found to be relatively small for the power level range under consideration (Gaussian beam $250 \mu \mathrm{m}$ with $\mathrm{P}_{\mathrm{opt}}=2 \mathrm{~W}$ to $8 \mathrm{~W}$ ). We found a cross-talk reduction factor of about $8 \%$ at $\mathrm{CW}$ illumination. The relative contribution of the non-linear thermal parameters to the total thermal cross-talk reduction decreases as the lock-in frequency increases, which results in a maximum deviation smaller than $1 \%$ at a lock-in frequency of $500 \mathrm{~Hz}$ for the power range under consideration. The relative importance of the individual non-linear thermal parameters taken into account by the model was studied by assuming constant values for all parameters, except for the one under investigation. The thermal conductivity $\mathrm{k}(\mathrm{T})$ of the active detector material was identified as the dominant parameter for the non-linearity assisted cross-talk reduction.

\section{Acknowledgements}

This work was funded by the Brussels region in the context of the SOIB project (BRGEOZ128) on opto-electronic semiconductor components for $\mathrm{CO}_{2}$ lasers and VUB (GOA51/DEFIS42012-VUB).

\section{References}

[1] Bauer J, Breitenstein O and Wagner J M 2009 Lock-in Thermography: A Versatile Tool for Failure Analysis of Solar Cells Electronic Device Failure Analysis 113 pp. 6-12.

[2] Vandermeiren W, Stiens J, De Tandt C, Shkerdin G, Kotov V, Borghs G, Muys P and Vounckx R 2010 Thermal cross-talk reduction in IR thermo-electric photodetectors by lock-in method: 4D numerical simulations and experimental validation. Proceedings of SPIE Photonics West - Physics and Simulation of Optoelectronic Devices XVIII, pp. 75970Y75970Y-9.

[3] Hanreich G, Nicolics J and Musiejovsky L 2000 High resolution thermal simulation of electronic components Microelectronics Reliability Elsevier Science 40 pp. 2069-76.

[4] Shkerdin, G.; Stiens, J. \& Vounckx, R. (1999). Comparative study of the intra- and intervalley contributions to the free-carrier induced optical nonlinearity in GaAs, J. Appl. Phys, Vol 87, 7, pp. 3807-18.

[5] Shkerdin G, Stiens J and Vounckx R 2002 X-valley influence on hot free electron absorption and nonlinearities at $10.6 \mu \mathrm{m}$ in highly doped GaAs European Phys. J. Appl. Phys, $191 \mathrm{pp}$. 29-38.

[6] Blakemore J S 1982 Semiconducting and other major properties of gallium arsenide, J. Appl. Phys. 5310 pp. R123-R181.

[7] Adachi S 1994 GaAs and related materials: Bulk Semiconductor and Supperlattice Properties (Singapore, World Scientific Publishing Co).

[8] Shkerdin G, Stiens J, Vandermeiren W, Kotov V, De Tandt C, Borghs G and Vounckx R 2006 $\mathrm{CO}_{2}$ laser induced temperature profiles in n-GaAs: an analytical model probed with the Seebeck effect J. Appl. Phys 100 (11) pp. 113120.

[9] George S N, Sharp J and Goldsmid H J 2001 Thermoelectrics: basic principles and new materials developments (Germany, Springer-Verslag Berlin Heidelberg).

[10] Burmeister L C 1993 Convective heat transfer, $2^{\text {nd }}$ ed. (Canada: John Wiley \& Sons, Inc.)

[11] Holman J P 1963 Heat transfer, $8^{\text {th }}$ ed. (USA: McGraw-Hill).

[12] Janicki M, Kindermann S, Pietrzak P and Napieralski A 2008 Estimation of local temperature dependent heat transfer coefficient for thermal analysis of electronic circuits Journal of Physics: Conference Series 135 012053doi:10.1088/1742-6596/135/1/012053.

[13] Le Houëdec H, Rochais D, Enguehard F, Jumel J and Lepoutre F (2005) Microscopic thermal characterization at temperatures up to $1000^{\circ} \mathrm{C}$ by photoreflectance microscopy J. Phys. D: Appl. Phys. 38, pp. 1498-1503.

[14] Walkey D J, Smy T J, MacElwee T, Maliepaard M 2001 Linear models for temperature and power dependence of thermal resistance in Si, InP and GaAs substrate devices. Semiconductor Thermal Measurement and Management. Seventeenth Annual IEEE Symposium, pp. 228-232. 
[15] Pipe K P, Ram R J, Shakouri A 2002 Bias-dependent Peltier coefficient and internal cooling in bipolar devices, Phys. Rev. B. 66, 125316.

[16] Hanreich G, Nicolics J 2001 Measuring the Natural Convective Heat Transfer Coefficient at the Surface of Electronic Components, IEEE Instrumentation and Measurement Technology Conference, pp. 1045-1050. 